\title{
Grain boundary relaxation in yellow gold bi-crystals
}

\author{
Ann-Kathrin Maier, Daniele Mari*, Iva Tkalcec, Robert Schaller \\ Ecole Polytechnique Fédérale de Lausanne, Institute of Condensed Matter Physics, Station 3, CH-1015 Lausanne, Switzerland
}

\section{A R T I C L E I N F O}

\section{Article history:}

Received 17 January 2015

Received in revised form

22 February 2015

Accepted 23 February 2015

Available online 3 March 2015

Keywords:

Grain boundaries

Bi-crystal

Internal friction

Gold alloy

Grain boundary sliding

\section{Introduction}

In metals, plastic deformation is achieved by dislocation motion. However, in polycrystalline specimens, a deformation between adjacent grains is often observed at high temperature. A number of studies testify the existence of grain boundary (GB) sliding, for example in ultrafine-grained $\mathrm{Cu}$ [1], in Au thin films [2], in AuPd [3] as well as in Pd [4] nanocrystalline materials. Direct observations of GB sliding have been made on single boundaries in Au nanopillars [5] and in $\mathrm{Zn}$ bi-crystals [6].

Mechanical spectroscopy is a non-destructive experimental technique that probes the mechanical response of a specimen to a periodic excitation. The technique is sensitive in particular to of GB sliding. A first model for GB sliding was developed by Zener in 1941 [7], which was verified experimentally by Kê [8] in polycrystalline Al. A GB peak in the mechanical loss spectrum has been observed in different polycrystalline materials [9-13]. This peak is absent in single crystalline specimens, which suggests that the relaxation peak is directly related to the presence of GBs.

A recent study on Au polycrystals [14] together with molecular dynamics simulations has shown that the microscopic stress relaxation mechanism acting at the GBs is probably due to GB sliding connected to a change in the GB structure. At elevated temperatures, the GB layer becomes disordered and a shearing parallel to the boundary plane is observed in the simulations.

In polycrystalline specimens, the experimental results reflect the mixed contributions from different types of GBs, which are characterized by distinct structures and properties [15]. In order to characterize

\footnotetext{
*Corresponding author. Tel.: +41 2169 34473; fax: +41216935459.

E-mail address: daniele.mari@epfl.ch (D. Mari).
}

the relaxation mechanism responsible for the mechanical loss peak, it is advantageous to study bi-crystals containing a single GB. Few studies on bi-crystals reporting the measurement of mechanical loss spectra are found in the literature, for example those related to pure $\mathrm{Cu}$ [16] and $\mathrm{Al}[17]$.

The investigations by Shi et al. $[18,17]$ on Al bi-crystals have been performed as a function of the misorientation angle showing that the activation parameters are distinct for high and low angle GBs. Furthermore, it was shown by Jiang et al. [19] that the relaxation strength of the $\mathrm{Al}$ bi-crystal's peak is proportional to the GB density, defined as the GB area per unit volume.

Up to now, mechanical spectroscopy measurements on bicrystals were made on pure metals. The present work shows that the mechanical loss peak of bi-crystals can also be observed in metallic alloys.

In this paper, we investigate the mechanical loss spectrum of a gold alloy bi-crystal as a function of different geometrical parameters such as the sample width, the thickness and the length, which account for different volume fractions with respect to the GB surface and for different stress distributions in the GB plane. The changes of the relaxation strength are compared with finite elements (FE) simulations, where we used the same sample dimensions as in the experiments. From the simulations, an empirical formula of the relaxation strength for rectangular sample geometries is derived, which can then be compared to the results of previous model calculations [14] assuming GB sliding at the origin of the stress relaxation mechanism.

\section{Experimental methods}

Bi-crystals of a commercial 18-carat (75 weight\%) gold alloy, which contains 30.5 atomic\% $\mathrm{Ag}$ and 9.9 atomic\% $\mathrm{Cu}$ were 


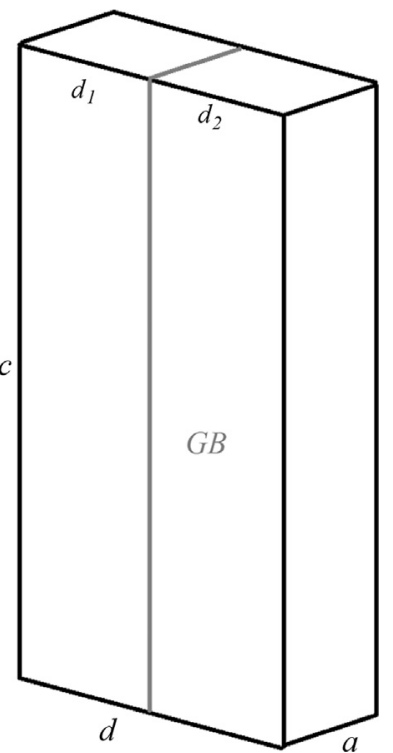

Fig. 1. Schematic drawing of the bi-crystal with vertical GB showing the width $d$, the thickness $a$ and the length $c$. The deformation axis is the vertical axis along $c$.

produced by the Bridgman technique. The sample was cut so that the GB is vertical in the torsion pendulum and the torsion axis lies in the GB plane. During the mechanical loss measurements, the lower sample end is fixed while the upper end is twisted periodically. The initial dimensions of the bi-crystal were $(12.3 \times 4.3 \times 0.78) \mathrm{mm}$. Fig. 1 shows the sample geometry. The length $c$, the width $d$ and the thickness $a$ were reduced step by step to observe in which way the loss spectrum of the bi-crystal depends on these parameters. The grain structure and the misorientation angle were controlled before and after the measurements. The misorientation angle remained stable at $\delta=(51.7 \pm 0.2)^{\circ}$. Tilt and twist components were present, characterizing this specific GB as a random boundary.

For comparison, a polycrystalline specimen was cut from a cylindrical wire of $2 \mathrm{~mm}$ in diameter. This sample had a homogeneous and small grained structure with an average grain diameter of $30 \mu \mathrm{m}$. A single crystal was grown in the same production step as the bi-crystal in the Bridgman oven. The bi-crystal's width was reduced by spark cutting, removing $1 \mathrm{~mm}$ of single crystalline material from the side. The left-over single crystal could be measured in the torsion pendulum.

The mechanical loss and the dynamic shear modulus have been measured in a forced torsion pendulum as a function of temperature at an imposed frequency of $f=0.5 \mathrm{~Hz}$ and at a constant strain amplitude of $1 \times 10^{-5}$. The heating and cooling rate was $2 \mathrm{~K} / \mathrm{min}$. In the case of an anelastic relaxation with a characteristic time $\tau$, the mechanical loss as a function of $\omega=2 \pi f$ takes the form of a Debye peak [20]:

$\tan \phi=\Delta \cdot \frac{\omega \tau}{1+\omega^{2} \tau^{2}}$

where $\Delta$ is the relaxation strength. The height of the Debye peak $\Delta / 2$ yields the relaxation strength, which is also defined as

$\Delta=\frac{\gamma_{a n}^{\infty}}{\gamma_{e l}}=\frac{\epsilon_{a n}}{\epsilon_{e l}}$

that relates the unrelaxed shear strain angle $\gamma_{e l}$ with the relaxed (anelastic) strain angle $\gamma_{a n}^{\infty}$. The maximum of the peak in Eq. (1) is obtained for $\omega \tau=1$. If the relaxation process is thermally activated, the relaxation time $\tau$ follows an Arrhenius equation: $\tau=\tau_{0} \exp \left(H_{a c t} /\left(k_{B} T\right)\right)$, where $\tau_{0}$ is the limit relaxation time and $H_{\text {act }}$ denotes the activation enthalpy.
If a peak in a mechanical loss spectrum appears broadened, one can assume that the relaxation time varies continuously around a mean value $\tau_{m}$ according to a log-normal distribution [21]:

$\Psi(z)=\frac{1}{\sqrt{\pi} \beta} \exp \left(-\frac{z^{2}}{\beta^{2}}\right) \quad$ with $z=\ln \left(\frac{\tau}{\tau_{m}}\right)$

The broadening is characterized by the distribution parameter $\beta$. Assuming that the relaxation strength $\Delta$ is identical for all values of $\tau$, one can calculate the analytic expression of the broadened Debye peak as a convolution of Eq. (1) with (3):

$\tan \phi=\frac{\Delta}{2 \sqrt{\pi} \beta} \int_{-\infty}^{\infty} \frac{\exp \left(-\frac{z^{2}}{\beta^{2}}\right)}{\cosh ((\ln \omega \tau)+z)} d z$.

A thermally activated and broadened Debye peak can be fully described by four parameters: the relaxation strength $\Delta$, the activation energy $H_{a c t}$, the limit relaxation time $\tau_{0}$ and the broadening factor $\beta$.

\section{Results}

Fig. 2 shows the mechanical loss spectrum and the dynamic shear modulus as a function of temperature of the polycrystal, the bi-crystal and the single crystal, which had been a part of the bi-crystal before. The mechanical loss shows peak P1 at around $600 \mathrm{~K}$ that may be attributed to a Zener relaxation due to stress induced diffusion of $\mathrm{Cu}$ atom pairs [22]. The P1 peak is present in all samples. Heating and cooling of the bi-crystal superimpose perfectly indicating that the microstructure is stable between room temperature and $950 \mathrm{~K}$. The spectra of the single crystal and the bi-crystal superimpose very nicely except for the peak P2. The polycrystal's spectrum is equally shown and its P2 peak is about twice as high and much broader.

Since the P2 peak is exclusively present in samples containing GBs, it can be concluded that the peak P2 is closely related to the presence of GBs. It can be ruled out that P2 is due to dislocation relaxation in the bulk or to a relaxation related to twin boundaries. The contribution of the bulk material is given by the spectrum of the single crystal and thus, the difference between the single crystal's spectrum and the bi-crystal's spectrum must be due to the grain boundary, which is the only structural defect that distinguishes single and bi-crystal.

From Fig. 2, it can be seen that the mechanical loss increases at high temperatures. However, in the fit of the complete spectrum, the high temperature background should be taken into

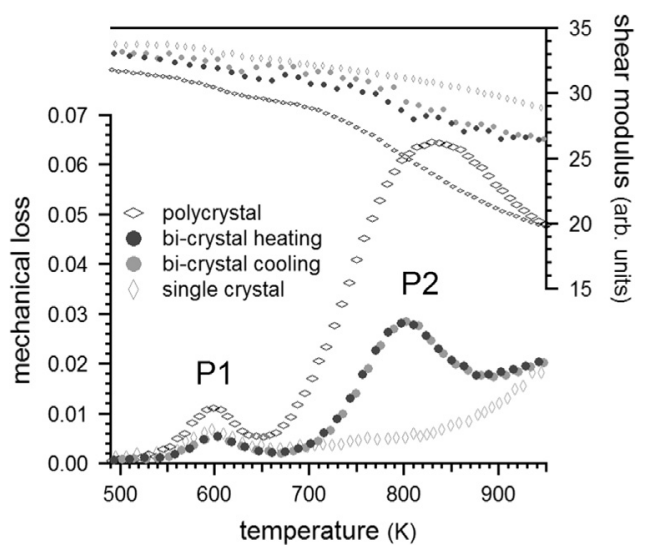

Fig. 2. Mechanical loss spectrum of the bi-crystal with a vertical GB. A relaxation peak accompanied by a modulus drop is observed at $800 \mathrm{~K}$. The spectrum of a polycrystal and a single crystal is shown for comparison. The single crystal has been cut from one side of the bi-crystal. 


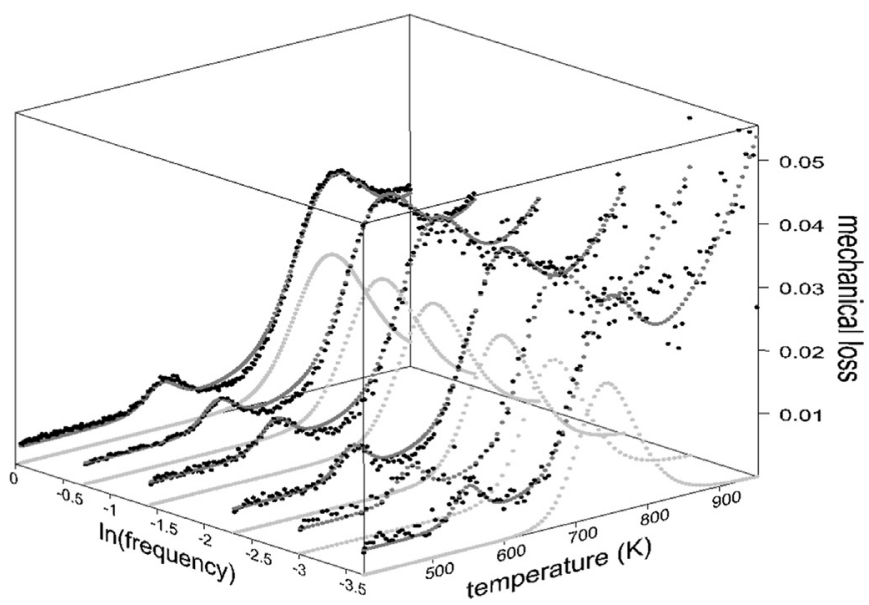

Fig. 3. Temperature scans of the bi-crystal (black markers) at different frequencies varying from $f=1 \mathrm{~Hz}$ to $f=0.025 \mathrm{~Hz}$. The fitted values are marked in dark gray, the contribution of the GB peak is shown in light gray. The fitting parameters are the same for all measurement points.

consideration. It has been shown by Schoeck [23] that an exponential background can be described by three parameters $K, n$ and $H_{B G}$ :

$\tan \phi=\frac{K}{(2 \pi f)^{n}} \exp \left(-\frac{n H_{B G}}{k_{B} T}\right)$

where $n$ is the broadening factor of the background and $K$ is the amplitude, which are valid over limited ranges of temperature.

Fig. 3 shows different temperature spectra measured at frequencies between $f=1 \mathrm{~Hz}$ and $f=0.025 \mathrm{~Hz}$ (black markers). Both peaks and the high temperature background are thermally activated, since they shift to higher temperatures with increasing frequency.

In order to extract the activation parameters of the bi-crystal, a different method than the usual Arrhenius method has been used, which gives a higher confidence level in the parameter values. For a data set of six temperature scans in Fig. 3, all data points have been fitted at once with the same parameter set. The fitting function is the sum of two Debye peaks (4) and an exponential background (5) plus a constant offset $a$ and an offset $b \cdot f$ accounting for experimental noise in the low frequency region. Since the relaxation strength $\Delta$ of the Zener peak P1 follows a Curie-Weiss behavior [24], $\Delta \propto\left(T-T_{c}\right)^{-1}$, and is therefore temperature dependent, we used the parameters for the Curie-Weiss constant $C$ and the ordering temperature $T_{C}$ instead of a constant $\Delta$.

All parameters are independent and the statistical errors reflect the correct uncertainties of the fitting parameters, since the parameters are all varied at the same time. The usage of classical analysis methods like Arrhenius plots and background subtractions can introduce a bias of the statistical errors, because a subset of the parameters is fitted. Then, the result is used to extract for example the activation energy in a second analysis step. The method used for this work does not suffer from this type of bias.

The fit of the bi-crystal's spectrum is shown in Fig. 3 with dark gray markers together with the contribution of the GB peak (light gray). Table 1 summarizes the values obtained for the parameters of P1 (first column), P2 (second column), the exponential background $\left(K, n\right.$ and $\left.H_{B G}\right)$ as well as the two offset parameters $a$ and $b$ using an offset of the form $a+b \cdot f$. The activation energy $H_{2}=(1.99 \pm 0.03) \mathrm{eV}$ for the GB peak in the bi-crystal is lower than the energy $H_{\text {poly }}=2.35 \mathrm{eV}$ [12] measured in a polycrystal of the same alloy. The limit relaxation time $\tau_{02}=(2.0 \pm 0.9) \cdot 10^{-13} \mathrm{~s}$ takes a perfectly reasonable value of the order of magnitude of the inverse Debye frequency, which, for a GB peak is not always the case. This is a further argument for the goodness of the parameter extraction method used here.

The broadening parameter $\beta=1.55 \pm 0.08$ is significantly lower than measured in the polycrystal $\left(\beta_{\text {poly }}=3.95 \pm 0.05\right)$ [25]. This can be easily understood, since the bi-crystal only contains a single type of GB, whereas many different GBs contribute to the relaxation peak in the polycrystal. The exponential background has the same activation energy $H_{B G}$ within the error bars as the P2 peak of the bi-crystal in Table 1 .

\subsection{Dependence on the geometrical parameters}

In general, mechanical loss peaks do not depend on the sample geometry but they depend on intrinsic parameters like the impurity content or the dislocation density. If the relaxation mechanism is highly stress dependent, the geometry can play a role since the distribution of the stress in the sample can vary with the sample shape. Other geometry dependencies can be due to the experimental set up. For example a temperature gradient along the sample length can cause a peak broadening. Using a shorter sample can reduce the gradient and the peak appears narrower.

The bi-crystal depicts a different behavior since the relaxation strength depends intrinsically on parameters like the sample width or the thickness. Since only one GB is present, the distribution of the defect (the GB), which causes a stress relaxation, is very inhomogeneous over the sample volume. A bi-crystal is very sensitive to the stress relaxation at the GB and it allows us therefore to test a different type of parameters, which is not possible in a homogeneous sample like a polycrystal.

The specimen geometry is shown in Fig. 1, where the names for the different sample dimensions have been chosen to be in agreement with the formulas in Ref. [14] and to permit an easy comparison of the experimental results with the theory.

Reducing the width $d$ by cutting the sample on both sides leads to a strong increase in the peak height (Fig. 4). In fact, it is not only the peak that increases, but the whole high temperature part of the spectrum. Fig. 4(b) shows the peak height of P2 as a function of the inverse width $1 / d$. It turns out [14] that the height and therefore the relaxation strength $\Delta$ is directly proportional to the grain boundary density $s=A / V=a c / a c d=1 / d$. A similar result has been found by Jiang et al. [19] in Al, where the authors showed that the GB peak height doubles when reducing the sample width by a factor of $1 / 2$.

Fig. 5(a) illustrates the dependence of $\Delta$ on the sample thickness $a$. It shows the inverse behavior as in the case of the width: when $a$ decreases, the peak height and the background decrease simultaneously. To reduce the width, the sample has been polished mechanically, in a first step from $0.78 \mathrm{~mm}$ to $0.64 \mathrm{~mm}$, which corresponds to a thickness reduction of $18 \%$ and in a second step to $0.54 \mathrm{~mm}$, which is $16 \%$ reduction. The background behaves the same way as the P2 peak, since the difference between the black and the dark gray curves continues on the high temperature part of the spectrum. Fig. 5(b) shows the peak height as a function of $a$ together with a linear fit through the origin. We observe that the peak height increases with $a$, even if the data points do not lie on a line. This shows that the peak height increases with the grain boundary width $a$. Changing $a$ does not affect the GB density $A / V=1 / d$.

As a third geometric parameter, the sample length dependence was tested on a bi-crystal having the GB centered as shown schematically in Fig. 1. The parameter $c$ has no influence on neither the peak height nor the position (Fig. 6). The slight shift of the $c=7.45 \mathrm{~mm}$ curve is more likely to be a measurement artefact than a real effect. In this case, the GB density is constant and a shearing in the vertical direction is suppressed, since the bicrystal is clamped at the top and at the bottom. 
Table 1

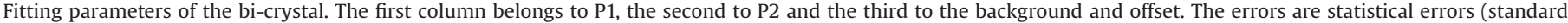
deviation with $1 \sigma$ confidence). In the case of $\tau_{01}$, the third column is the upper limit with $2 \sigma$ confidence.

\begin{tabular}{|c|c|c|c|c|c|c|c|c|}
\hline & \multicolumn{2}{|l|}{ P1 } & & \multicolumn{2}{|l|}{ P2 } & & \multicolumn{2}{|l|}{ BG } \\
\hline & Value & Error & & Value & Error & & Value & Error \\
\hline$C(\mathrm{~K})$ & 1.8 & 0.5 & $\Delta_{2}$ & 0.060 & 0.002 & K & 11 & 2 \\
\hline$T_{c}(\mathrm{~K})$ & 400 & 50 & $\tau_{02}(\mathrm{~s})$ & $2.0 \times 10^{-13}$ & $9 \times 10^{-14}$ & $n$ & 0.239 & 0.007 \\
\hline$\tau_{01}(\mathrm{~s})$ & $5 \times 10^{-17}$ & $<2 \times 10^{-16}$ & $\mathrm{H}_{2}(\mathrm{eV})$ & 1.99 & 0.03 & $H_{\mathrm{BG}}(\mathrm{eV})$ & 2.01 & 0.05 \\
\hline$H_{1}(\mathrm{eV})$ & 1.88 & 0.14 & $\beta_{2}$ & 1.55 & 0.08 & $a$ & 0.0039 & 0.0002 \\
\hline$\beta_{1}$ & 0.9 & 0.4 & & & & $b$ & -0.0013 & 0.0002 \\
\hline
\end{tabular}
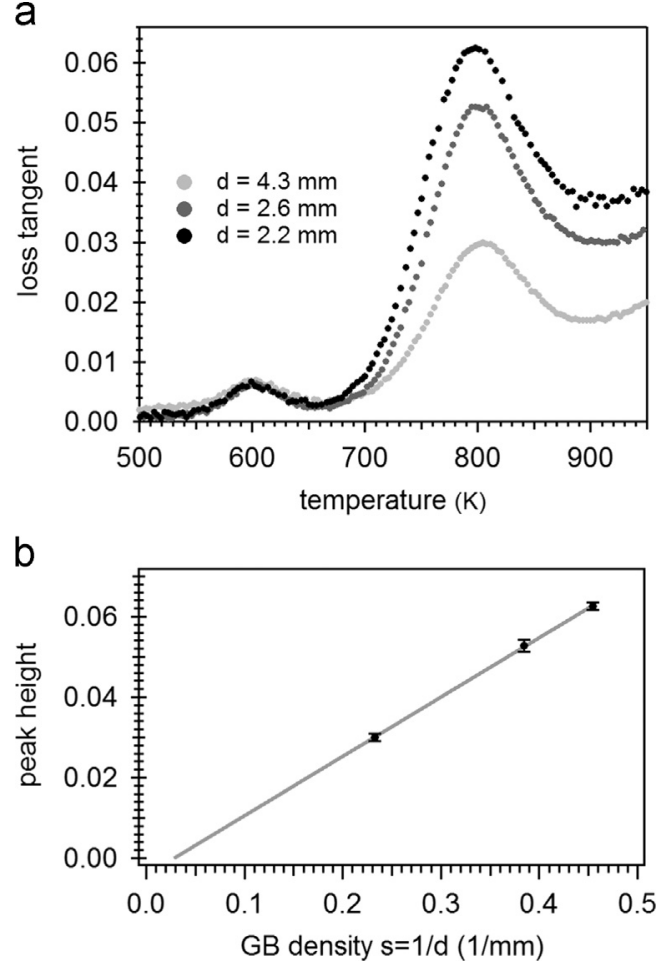

Fig. 4. (a) Dependence of the bi-crystal's peak on the width $d$. The peak height increases with decreasing $d$. The background changes in the same way. The peak position remains unchanged. (b) The peak height as a function of the inverse width $1 / d$ shows a linear relationship.

The last geometrical parameter, on which a dependence of the GB peak height was observed, is an asymmetry factor $\left|d_{1}-d_{2}\right| / d$, where $d_{1}$ and $d_{2}$ are the widths of the two single crystalline parts in Fig. 1. This parameter varies between 0 and 1, where a centered GB has the value 0 and a completely off-centered GB has a value close to 1.

A dependence on the asymmetry factor has been observed only qualitatively. A sample with a curved GB illustrated in Fig. 7(a) has been measured as a function of the length $l$. Since the peak of a bicrystal with a centered GB does not depend on the length, the only parameter, which changes in the curved GB sample, is the mean position of the GB. Fig. 7(b) shows that the GB peak decreases in height when the GB is shifted to the sample border. The background on the other hand remains unchanged except for the shortest sample with $l=7.3 \mathrm{~mm}$.

\section{Discussion}

In Ref. [26], it has been shown that the GB peak of a polycrystal depends on the grain size $d$. More precisely, with higher $d$, the GB
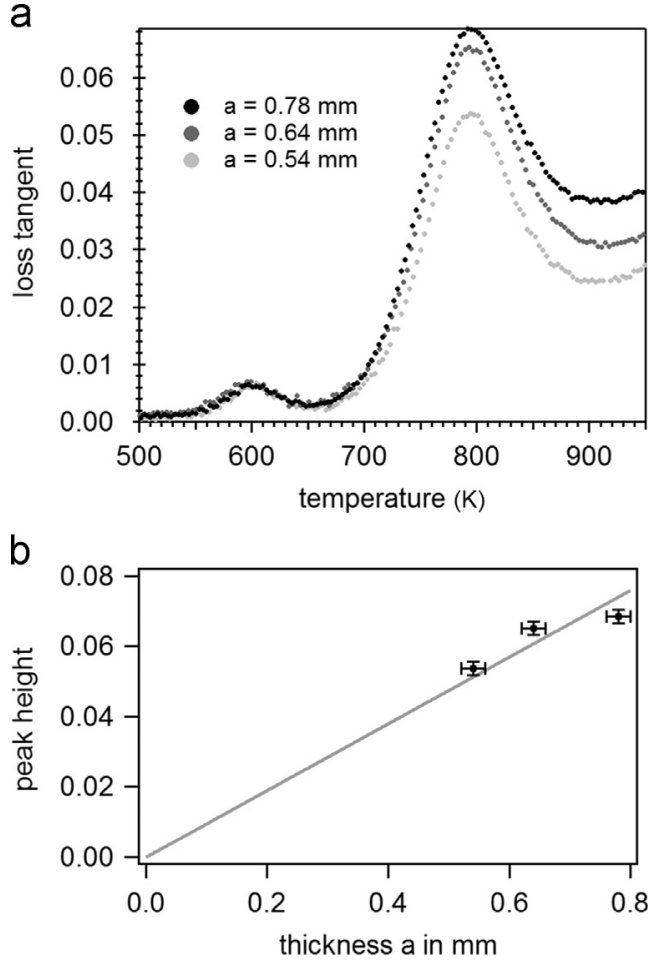

Fig. 5. (a) Mechanical loss spectrum of the bi-crystal with different thickness $a$. A reduction of $a$ by polishing leads to a decrease of peak P2 and the background. (b) Peak height as a function of the thickness $a$. The gray line is only a guide for the eyes.

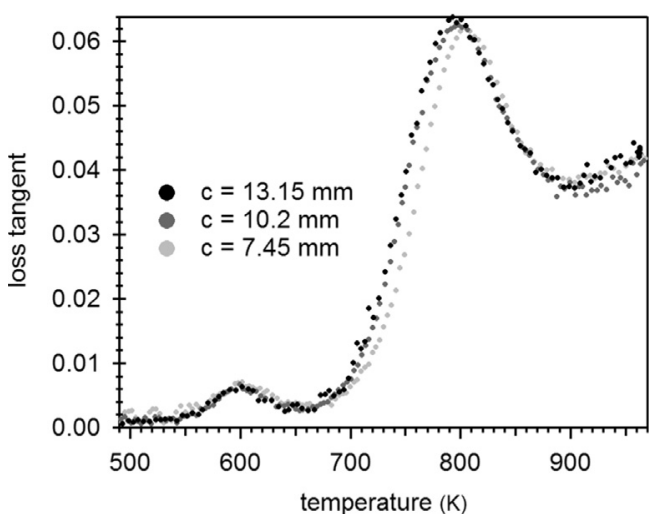

Fig. 6. Spectrum of the bi-crystal with varying clamping lengths. The mechanical loss does not depend on the parameter $c$ in the case of a centered GB.

peak is observed at higher temperatures, whereas the peak height and shape remain unchanged. The observed linear relationship between $\tau$ and $d$, i.e. $\tau \propto d$ and therefore a grain size exponent equal to 1, is in full agreement with the Zener model [7] of grain 


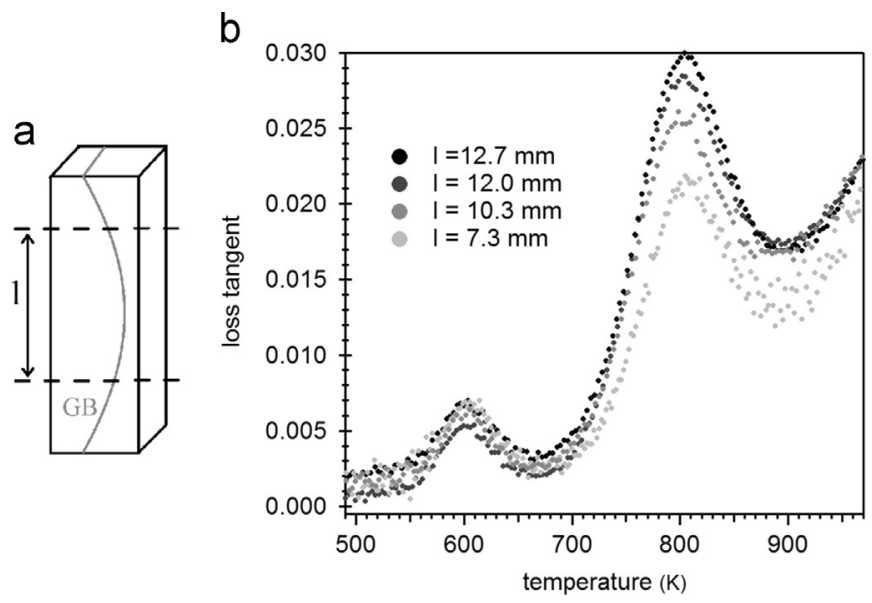

Fig. 7. (a) Schematic drawing of a bi-crystalline sample with a shifted and curved grain boundary. A reduction of the length shifts the mean position to the right side. (b) Temperature spectra of the bi-crystal at different lengths $l$, which is more a parameter for the shift of the GB to one side. The GB peak decreases when the mean GB position is shifted to the border of the sample.

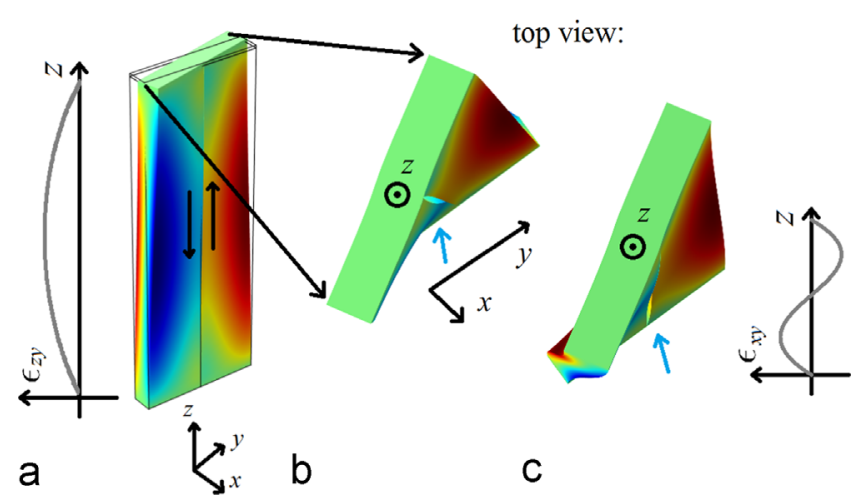

Fig. 8. (a) FE simulations of a sample with central GB. The deformation is enhanced by a factor of 3200 . The colors on the surface indicate the displacement in the $z$ direction, where red zones have moved upwards and blue zones downwards. The color is discontinuous along the boundary plane indicating a glide. The effect is most pronounced in the middle of the sample and tends to 0 on to and on the bottom. (b) and (c) Top view of the same sample: the green surface is the top surface and the curved line belongs to the GB. A sliding along the $x$-axis occurred with different directions in the top half (light blue surface in $\mathrm{b}$ ) and the bottom half (yellow surface in c). No sliding in the $x$-direction is observed in the center of the sample. The displacement is enhanced by a factor of 8000 . (For interpretation of the references to color in this figure caption, the reader is referred to the web version of this paper.)

boundary sliding in a polycrystal. If we make the hypothesis that GB sliding on a microscopic scale has a macroscopic effect as if two elastic solids can glide along their common surface, we can perform finite element (FE) simulations to test the geometric dependencies.

\subsection{FE simulations}

The simulations were performed on two vertical bars, which are joined to form a bi-crystal with a flat sliding surface in order to check, if the geometry dependencies observed in the mechanical loss experiment are linked to the intrinsic GB type with its microscopic structure or to the stress distribution in the sample. Along the GB, the material can slide with respect to the adjacent material without friction. The GB is infinitely thin and we do not consider that the misorientation or the plane direction with respect to the crystal lattice could influence the GB structure.
The only parameter that is probed in these simulations is the relaxation of local stresses in the vicinity of the GB. The simulations used gold as a bulk material and the geometry and forces were chosen according to the experimental set-up shown in Fig. 1. As in the experiment, a torque is applied along the vertical axis $c$.

In the experiment, the relaxation strength $\Delta$ is given by the peak height (1), but in principle it could also be measured in a static deformation experiment. The relaxation strength is given by Eq. (2): $\Delta=\epsilon_{a n} / \epsilon_{e l}$. In the FE simulation, $\Delta$ can be calculated from the deformation of a sample without GB and with GB. At a constant force, $\epsilon_{e l}$ is given by the maximum deformation of a continuous sample without GB. $\epsilon_{a n}$ can be extracted from the difference $\epsilon_{\text {tot }}-\epsilon_{e l}$, where $\epsilon_{\text {tot }}$ is the maximum deformation of the sample with a GB.

For each sample geometry, the force on the top surface was adapted, such that the total strain $\epsilon_{\text {tot }}$ reached $10^{-5}$, since the experiments were also performed in constant strain mode with $10^{-5}$ as a maximal strain amplitude.

The introduction of a vertical GB leads to a stress relaxation along the GB in the FE simulations. Fig. 8(a) and (b) visualizes two different sliding modes that occur at the GB. Fig. 8(a) shows the $z$-displacement in the presence of a GB. The thin black lines show the sample position without deformation and the surface color illustrates the displacement in the z-direction. Mainly the vertical edges show a displacement upwards (red) or downwards (blue). The coloring is discontinuous at the boundary. The right side slides upwards with respect to the left side. A shear component $\epsilon_{z y}$ is observed at the GB plane, where the first index corresponds to the direction of the shear displacement and the second index is the normal direction of the shear plane. The small diagram on the left of the colored bi-crystal shows that the sliding amount is highest in the middle of the GB.

The grain boundary also slides along the perpendicular $x$-axis, as it can be seen from Fig. 8(b) and (c). The sample is viewed from above with the green surface being the bi-crystal's top surface. The grain boundary is seen as a curved line, where a relative displacement of both sample parts occurred. The sliding in the top half is opposite to the sliding in the bottom half. A diagram on the right of Fig. 8(c) shows qualitatively the shear component $\epsilon_{x y}$.

The shear along $x$ and $z$ together can be seen as a rotational shear around the $y$-axis. The right part of the sample colored in red turns in the negative $y$-direction, whereas the left part turns in the positive $y$-direction. On a local scale, the shear stress varies along the GB plane and has different directions in form of a simple shear component.

Fig. 9 shows the quantitative results of the FE simulations, where the relaxation strength has been measured for various sample geometries. The absolute relaxation strength is much higher than the one measured in a real bi-crystal, since the FE model has a completely flat boundary and the gliding is frictionless.

Observations of grain boundaries by electron microscopy in transmission in $\mathrm{Al}[15]$ and in $\mathrm{Au}[27,28]$ show a crystalline GB structure with a zig-zag profile at room temperature, which becomes flatter upon heating. Even if the GB is flat at the atomic scale at temperatures where GB sliding becomes active, the GB is probably not flat at macroscopic scales over the whole sample. The perfect flatness of the GB in the FE simulations is a simplification and in the real GB has a certain roughness, which leads to an additional contribution of the restoring force. Therefore, the relaxation strength caused by a real GB is expected to be much smaller than the simulated relaxation strength.

The FE simulations are in general agreement with the experimental findings from mechanical spectroscopy measurements. $\Delta$ varies very slightly with the sample length $c$ both for a centered GB and a GB shifted to one side so that $d_{1}=3 \cdot d_{2}$. In both cases, the GB plane is a perfectly flat surface. As in the experiments, the simulated relaxation strength is proportional to the thickness $a$ 

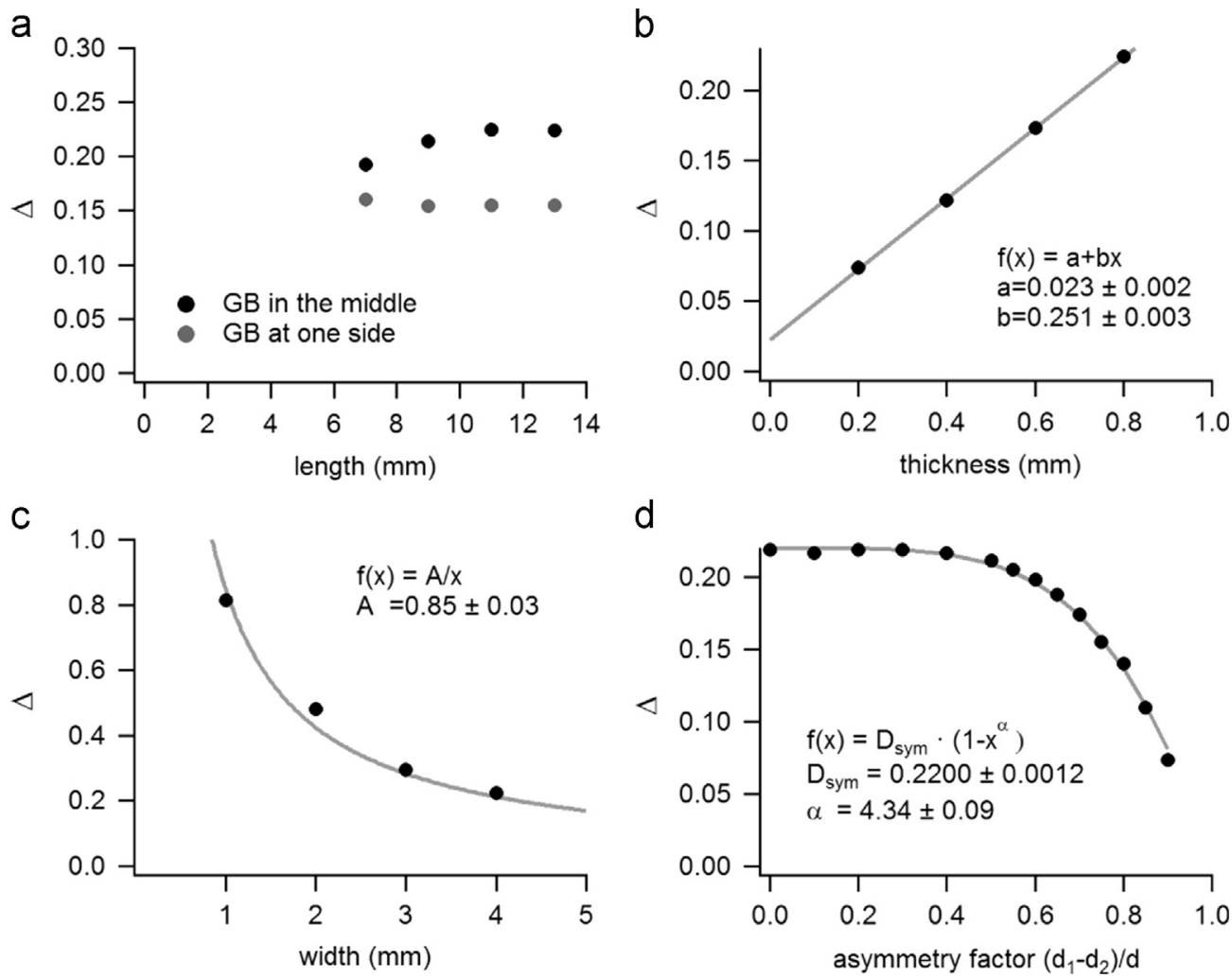

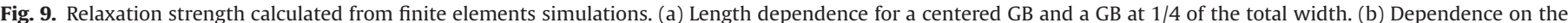

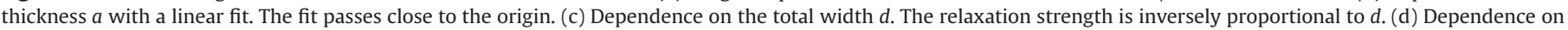
the asymmetry factor $\left|d_{1}-d_{2}\right| / d$. $\Delta$ decreases for an off-centered boundary. The parameter $D_{\text {sym }}$ is the relaxation strength of a symmetric boundary plane.

(Fig. 9(b)) and inversely proportional to the width $d$ (Fig. 9(c)). If the GB is shifted to one side of the sample, the relaxation strength decreases. These geometric dependencies can be summarized in a phenomenological formula:

$\Delta=\Delta_{0} \cdot \frac{a}{d}\left(1-\left(\frac{\left|d_{1}-d_{2}\right|}{d}\right)^{\alpha}\right)$.

$\Delta_{0}$ is the relaxation strength for a centered GB in a rectangular sample with square base $a=d$. The simulated data set provides values $\Delta_{0} \approx 1.1$ and $\alpha=4.34 \pm 0.09$. A comparison with the experimental findings shows a qualitative agreement with Eq. (6). The parameter $\alpha$ cannot be extracted from the experimental data, since we do not know the exact correlation between the clamping length $l$ and the mean GB position for the curved GB.

The interpolation of Fig. 4(b) for a sample with square base $a=d=0.78 \mathrm{~mm}$ leads to $\Delta_{0}=0.189$, which is six times smaller than the value from the FE simulations. The difference between simulation and experiment indicates that friction plays an important role when the microscopic mechanism responsible for the GB peak is related to GB sliding.

The experiments show a mechanical loss spectrum with two peaks and a high temperature background. When the sample thickness or the width was changed (Figs. 4a and 5a), not only did the peak height change but also the background.

It has been shown in Ref. [25] that the background increases when the dislocation density in a single crystal is increased. Therefore, at least a part of the high temperature background should be due to a dislocation relaxation. On the other hand, the correlation between the GB peak and the background is quite obvious in the bi-crystal spectra. The background increases in the same manner as the GB peak, when the width $d$ is reduced. If the background were only due to dislocations in the single crystalline parts, it would be difficult to understand why the background should increase when a part of the single crystalline material is removed. It seems that at least a part of the background in the bi-crystals is originating from the same relaxation mechanism that causes the GB peak.

Morris and Jackson [29] presented an analysis of the Raj/Ashby model [30] of GB sliding, where energy dissipation is caused by two mechanisms: boundary sliding and GB diffusion. If the characteristic time scales for both mechanisms are sufficiently distinct, the mechanical loss spectrum presents a low frequency (high temperature) background as well as a mechanical loss peak. The peak is due to a viscous sliding along the GBs, whereas the background is due to GB diffusion. Taking only the diffusional term into consideration, the model predicts a steady state creep for low frequencies (high temperature).

From the mechanical loss spectrum in polycrystals, the diffusional mechanism producing a local creep at the GB can be understood in the following way. The polycrystal spectrum shows a relaxation peak due to GB sliding. The restoring force due to the presence of adjacent grains is supposed to be temperature independent and at high temperatures or low frequencies, a perfect relaxation peak should tend to 0 . If, on the other hand, the pinning points responsible for the restoring force start to weaken at high temperatures, the restoring force itself decreases and one should observe the onset of local creep. In the mechanical loss spectrum, the relaxation peak transforms into a high temperature background instead of decreasing to 0 .

Therefore, the high temperature background is probably caused by two different mechanisms. In samples that contain GBs, a part of the background is due to GB diffusion, superimposed on the background caused by dislocations in the bulk.

\section{Conclusions}

The mechanical loss spectrum of yellow gold alloy polycrystals is mainly composed of two relaxation peaks and a high temperature 
exponential background. The first peak (P1), which is observed at about $600 \mathrm{~K}$ for a $0.5 \mathrm{~Hz}$ frequency, is due to a Zener type anelastic relaxation.

The second peak (P2), which appears in polycrystals and in bicrystals, but not in single crystals, is due to a relaxation associated with the GBs. The peak observed in bi-crystals is narrower than in a polycrystal and its height depends on external geometrical parameters like the sample thickness and the width. The relaxation strength is inversely proportional to the width $d$, which means that the relaxation strength scales with the volume fraction of GBs. $\Delta$ is proportional to the sample thickness $a$, which in the present model is the thickness of the GB plane. Furthermore, the relaxation strength is independent of the length $l$, since the sample is fixed in this direction. If the GB in the bi-crystal is situated close to one side, the relaxation strength is decreased.

Finite Elements simulations can reproduce the qualitative change of the relaxation strength with different sample geometries. An expression of the relaxation strength as a function of the geometrical parameters $a, d, l$ and the asymmetry factor $\left|d_{1}-d_{2}\right| / d$ has been developed. It is therefore possible to separate the geometrical part of the relaxation strength from the microscopic part.

\section{Acknowledgments}

This work was financially supported by the Swiss National Science Foundation (Grant no. 200020_143913/1). Varinor SA is acknowledged for gold alloy supply.

\section{References}

[1] I.S. Golovin, P. Pal-Val, L. Pal-Val, E. Vatazhuk, Y. Estrin, Solid State Phenom. 184 (2012) 289-294.
[2] G.-D. Sim, J.J. Vlassak, Scr. Mater. 75 (2014) 34-37.

[3] W. Skrotzki, A. Eschke, B. Jóni, T. Ungár, L. Tóth, Y. Ivanisenko, L. Kurmanaeva, Acta Mater. 61 (2013) 7271-7284.

[4] K. Yang, H.-J. Fecht, Y. Ivanisenko, Adv. Eng. Mater. 16 (2014) 517-521.

[5] F. Lançon, J. Ye, D. Caliste, T. Radetic, A.M. Minor, U. Dahmen, Nano Lett. 10 (2010) 695-700 20143874.

[6] A. Sheikh-Ali, Scr. Metall. Mater. 33 (1995) 795-801.

[7] C. Zener, Phys. Rev. 60 (1941) 906-908.

[8] T.-S. Ke, Phys. Rev. 72 (1947) 41-46.

[9] E. Bonetti, A. Cavallini, E. Evangelista, P. Gondi, J. Phys. Colloq. 44 (1983) 759-764.

[10] W. Benoit, Mater. Sci. Eng. A 370 (2004) 12-20.

[11] A. Isoré, W. Benoit, P. Stadelmann, Philos. Mag. 34 (1976) 811-838.

[12] A.K. Maier, I. Tkalcec, D. Mari, R. Schaller, Solid State Phenom. 184 (2012) 283-288.

[13] I. Tkalcec, J. Hennig, D. Mari, R. Schaller, Mater. Sci. Eng. A 521-522 (2009) 52-54.

[14] A.-K. Maier, D. Mari, I. Tkalcec, R. Schaller, Acta Mater. 74 (2014) 132-140.

[15] A. Sutton, R. Balluffi, Interfaces in Crystalline Materials, Monographs on the Physics And Chemistry of Materials, OUP, Oxford, 1995.

[16] M. Kato, T. Mori, Philos. Mag. A 68 (1993) 939-949.

[17] Y. Shi, W.B. Jiang, Q.P. Kong, P. Cui, Q.F. Fang, M. Winning, Phys. Rev. B 73 (2006) 174101.

[18] Y. Shi, P. Cui, Q.P. Kong, W.B. Jiang, M. Winning, Phys. Rev. B 71 (2005) 060101 (R).

[19] W. Jiang, Q. Kong, P. Cui, Mater. Sci. Eng.: A 527 (2010) 6028-6032.

[20] A.S. Nowick, B.S. Berry, Anelastic Relaxation in Crystalline Solids, Academic Press, New York, 1972.

[21] R.M. Fuoss, J.G. Kirkwood, J. Am. Chem. Soc. 63 (1941) 385-394.

[22] J. Hennig, D. Mari, R. Schaller, Phys. Rev. B 79 (2009) 144116.

[23] G. Schoeck, E. Bisogni, J. Shyne, Acta Metall. 12 (1964) 1466-1468.

[24] J. Hennig, D. Mari, R. Schaller, Mater. Sci. Eng. A 521-522 (2009) 47-51.

[25] A.-K. Maier, I. Tkalcec, D. Mari, R. Schaller, Mater. Sci. Eng.: A 560 (2013) 466-472.

[26] A.-K. Maier, I. Tkalcec, D. Mari, R. Schaller, Scr. Mater. 66 (2012) 374-377.

[27] R.C. Pond, S. Celotto, Int. Mater. Rev. 48 (2003) 225-245.

[28] T.E. Hsieh, R.W. Balluffi, Acta Metall. 37 (1989) 2133-2139.

[29] S. Morris, I. Jackson, J. Mech. Phys. Solids 57 (2009) 744-761.

[30] R. Raj, M.F. Ashby, Metall. Mater. Trans. B 2 (1971) 1113-1127. 